\title{
Sensory classification of table olives using an electronic tongue: Analysis of aqueous pastes and brines
}

\author{
Itala Ítala Marx ${ }^{\mathrm{a}, \mathrm{b}}$, Nuno Rodrigues ${ }^{\mathrm{c}, \mathrm{d}}$, Luís G. Dias ${ }^{\mathrm{a}, \mathrm{e}}$, Ana C.A. Veloso ${ }^{\mathrm{f}, \mathrm{g}}$, José A. Pereira ${ }^{\mathrm{h}}$, \\ Deisy A. Drunkler ${ }^{\mathrm{b}}$, António M. Peres ${ }^{\mathrm{i}, *}$ \\ a School of Agriculture, Polytechnic Institute of Bragança, Campus Santa Apolónia, 5300-253 Bragança, Portugal \\ b Universidade Tecnológica Federal do Paraná-UTFPR, Avenida Brasil, Câmpus Medianeira, 4232-Parque Independência, Medianeira, Parana 85884-000, \\ Brazil \\ ${ }^{\mathrm{c}}$ REQUIMTE-LAQV/CIMO, School of Agriculture, Polytechnic Institute of Bragança, Campus Santa Apolónia, 5300-253 Bragança, Portugal \\ d Universidad de Léon, Departamento de Ingeniería Agrária, Av. Portugal, no. 41, 24071 Léon, España \\ e CQ-VR, Centro de Química - Vila Real, University of Trás-os-Montes e Alto Douro, Apartado 1013, 5001-801 Vila Real, Portugal \\ ${ }^{\mathrm{f}}$ Instituto Politécnico de Coimbra, ISEC, DEQB, Rua Pedro Nunes, Quinta da Nora, 3030-199 Coimbra, Portugal \\ g CEB - Centre of Biological Engineering, University of Minho, Campus de Gualtar, 4710-057 Braga, Portugal \\ ${ }^{\mathrm{h}}$ REQUIMTE-LAQV, School of Agriculture, Polytechnic Institute of Bragança, Campus Santa Apolónia, 5300-253 Bragança, Portugal

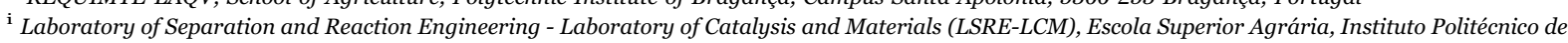 \\ Bragança, Campus Santa Apolónia, 5300-253 Bragança, Portugal
}

\section{A R T I C L E I N F O}

\section{Keywords:}

Electronic tongue

Table olives

Sensory defects

Trade category classification

Linear discriminant analysis

Simulated annealing algorithm

\begin{abstract}
A B S T R A C T
Table olives are highly appreciated and consumed worldwide. Different aspects are used for trade category classification being the sensory assessment of negative defects present in the olives and brines one of the most important. The trade category quality classification must follow the International Olive Council directives, requiring the organoleptic assessment of defects by a trained sensory panel. However, the training process is a hard, complex and sometimes subjective task, being the low number of samples that can be evaluated per day a major drawback considering the real needs of the olive industry. In this context, the development of electronic tongues as taste sensors for defects' sensory evaluation is of utmost relevance. So, an electronic tongue was used for table olives classification according to the presence and intensity of negative defects. Linear discrimination models were established based on sub-sets of sensor signals selected by a simulated annealing algorithm. The predictive potential of the novel approach was first demonstrated for standard solutions of chemical compounds that mimic butyric, putrid and zapateria defects ( $\geq 93 \%$ for cross-validation procedures). Then its applicability was verified; using reference table olives/brine solutions samples identified with a single intense negative attribute, namely butyric, musty, putrid, zapateria or winey-vinegary defects ( $\geq 93 \%$ cross-validation procedures). Finally, the E-tongue coupled with the same chemometric approach was applied to classify table olive samples according to the trade commercial categories (extra, $1^{\text {st }}$ choice, $2^{\text {nd }}$ choice and unsuitable for consumption) and an additional quality category (extra free of defects), established based on sensory analysis data. Despite the heterogeneity of the samples studied and number of different sensory defects perceived, the predictive linear discriminant model established showed sensitivities greater than $86 \%$. So, the overall performance achieved showed that the electrochemical device could be used as a taste sensor for table olives organoleptic trade successful classification, allowing a preliminary quality assessment, which could facilitate, in the future, the complex task of sensory panelists.
\end{abstract}

\section{Introduction}

Table olives are a food product highly appreciated by consumers [1] and recognized as a source of bioactive compounds [2], and their commercialization represent substantial financial revenue. It is of major relevance to guarantee the quality and safety of the final product, which requires monitoring physicochemical, microbiological and sensory parameters of olives and brine solutions along the production, packing and storage chain [3]. Physicochemical, microbiological and sensory evaluations must be carried out following the methodologies,

\footnotetext{
* Corresponding author

E-mail address: peres@ipb.pt (A.M. Peres).
} 
grading systems and procedures established by international regulations $[4,5]$. The analysis allows classifying table olives according to different grading scales, namely inedible (unsuitable for consumption) or edible (suitable for consumption). Table olives prices and consumers' preferences will greatly depend on their trade category. Table olives may be classified according to olive sizes (e.g., bullets, fine, superior, extra large, jumbo, colossal, etc.), physical quality criteria (extra or fancy, first choice or select olives and second choice or standard olives) or organoleptic grading based on the median intensity of the defect predominant perceived (DPP) by a trained sensory panel during the analysis of both olives and brine solutions (extra, first choice, second choice and olives that cannot be sold as table olives) $[3,6]$, as recommended by the International Olive Council (IOC) [5]. This latter commercial classification, although recommended and implemented already by several table olive producers, is not yet legally required. Panelists must evaluate olfactory defects (e.g., abnormal fermentation, which includes butyric, putrid and "zapateria" sensations) as well as olfactory-gustatory negative attributes (e.g., musty; rancid; cooking effect; soapy; metallic; earthy and winey-vinegary sensations). Sensory evaluation of table olives performed by trained panels is still quite applied [7-21], although it is not always feasible, mainly due to the scarcity of trained sensory panels, the cost and the time required for analysis, as well as due to the low number of samples that can be daily evaluated [22].

So, there is a clear practical need to develop alternative or complementary analytical methods that could allow the organoleptic assessment of table olives (olives and brine solutions). Some chromatographic based methods have been reported for assessing sensory attributes of table olives $[23,24]$. However, these methodologies are time-consuming, require sophisticated equipment and skilled technicians [22]. Other instrumental techniques (e.g., headspace-mass spectrometry, mid-infrared spectroscopy and UV-visible spectrophotometry) fused with multivariate analysis showed to be a useful tool to classify olive oil samples based on their category and the presence of certain sensory defects [25]. Nevertheless, it is of utmost interest to investigate the potential of low-cost, fast and sensitive analytical procedures to evaluate the overall quality of table olives. Recently, electronic tongues (E-tongue) and/or electronic noses (E-noses) have been proposed for sensory and physico-chemical characterization of olive oils [26,27]. Panagou et al. [22] demonstrated the capability of an E-nose as a screening tool for quality control of fermented table olives based on their volatile patterns. The device enabled to discriminate green olives according to three major classes (acceptable, unacceptable and marginal) based on the evaluation of a sensory panel. The present work aims to evaluate, for the first time, the possibility of applying a potentiometric E-tongue combined linear discriminant analysis (LDA) and meta-heuristic simulated annealing (SA) variable selection algorithm, to: (i) identify and discriminate the most common negative sensory attributes of table olives; and (ii) to classify table olives according to their trade commercial quality classification [6]. For that, first the E-tongue capability to evaluate common negative organoleptic attributes due abnormal fermentation (i.e., butyric, putrid and zapateria) was evaluated using standard solutions within the concentration ranges recommend by IOC during the training of a sensory panel [6]. Then, the capability of the E-tongue to distinguish, separately, selected samples of table olives or brine solutions, for which a single predominant negative attribute (e.g., butyric, putrid and zapateria, wineyvinegary or musty defects) was perceived by a trained sensory panel [6]. Finally, the possibility of applying the E-tongue for assessing table olives trade quality category (previously established by the sensory panel considering the median intensity of the defect predominant perceived (DPP) in the tables olives and/or the respective brine solutions) was evaluated by using simultaneously the signal profiles recorded for table olives (aqueous diluted olive paste) and brine solutions, mimicking the usual classification procedure followed during the sensory analysis.

\section{Materials and methods}

\subsection{Table olives samples}

Forty four table olives commercial samples from different brands (18 brands) were purchased in local supermarkets in Bragança (Portugal) and Zamora (Spain), being 36 obtained from natural fermentation (aromatized with spices or not), 6 are Spanish-style (with or without added flavors), 1 is California-style (no aromatized) and 1 aromatized from mixed styles (natural fermentation, Spanish-style and California-style). Furthermore, the olives used in the production processes were from 7 Portuguese or Spanish cultivars namely cvs Cobrançosa, Galega, Gordal, Empeltre, Negrinha de Freixo, Hojiblanca and Manzanilla. The samples were stored in the original packaging, in dark at ambient temperature $\left(\sim 20^{\circ} \mathrm{C}\right)$ until analysis or after opened in the refrigerator at $4{ }^{\circ} \mathrm{C}$ (being all of them analyzed before the end of the expiration date). It should be remarked that the number of different independent samples studied is of the same order of magnitude as those usually used in the literature regarding table olives analysis [1720,22,28-30].

\subsection{Table olives organoleptic analysis: sensory panel and sample preparation}

The organoleptic evaluation of the table olives and respective brine solutions was performed by a trained sensory panel, formed by eight selected panelists that worked or studied at the School of Agriculture of the Polytechnic Institute of Bragança (Portugal), including individuals of both sexes and different ages (from 22 to 56 years). The training process included theoretical and practical sessions held twice a week and that followed the main recommendations of the IOC standard regulation [6]. The sensory panel was trained during 18 months, before performing the analysis reported in this work.

Sensory analysis of table olives involves, among other, the detection of negative attributes (e.g., butyric, putrid and "zapateria" due to abnormal fermentation; and other defects like winey-vinegary, musty, cooking effect, rancid, etc.) and the assessment of the respective intensities. The intensities are scaled from 1 (lowest intensity, attributed when a defect is not perceived) to 11 (highest intensity) [6]. The type and intensity of the negative sensory attributes present in the table olives (olives and brine solutions) are the sole attributes used for commercially classifying the sensory quality of table olives [6]. The trade category classification is based on the median intensity perceived by the panel for the DPP. The table olive samples can be classified as extra (DPP $\leq 3)$, first choice $(3<\mathrm{DPP} \leq 4.5)$, second choice $(4.5<$ $\mathrm{DPP} \leq 7.0$ ) or table olives that cannot be sold as table olives (DPP $>$ 7.0). In this work, an additional category was taken into account for classification purposes (extra table olives free of any defect; extra_wd), including the table olives for which no organoleptic defect could be perceived by any member of the sensory panel (DPP $=1$ ). The inclusion of this new category relayed on the findings reported by Lanza and Amoruso [17], according to which the occurrence of a sensory defect changes the entire organoleptic profile of the table olives being samples classified as "Extra or Fancy", with DPP greater than 1.0, quite similar to samples with more intense negative attributes. The statistical data treatment was carried out using the official software (CALC-ENG V08 01-06-14 IOC TABLE OLIVE) provided by IOC [6]. For the sensory evaluation, the table olive samples were prepared following the guidelines of the official regulation IOC/OT/MO No 1/Rev.2 November 2011 $[6]$.

\subsection{E-tongue device}

The E-tongue multi-sensor device included two print-screen potentiometric arrays ( $9.5 \mathrm{~cm}$ of width and $2.5 \mathrm{~cm}$ of height) containing each one 20 sensors ( $3.6 \mathrm{~mm}$ of diameter and $0.3 \mathrm{~mm}$ of thickness) 
Table 1

E-tongue sensors details ( sensor identification code, plasticizer and additive compounds used in the preparation of each lipid-polymeric membrane).

\begin{tabular}{llll}
\hline No. & \multicolumn{2}{c}{ Plasticizer } & Additive \\
\hline S1:1 & S2:1 & 2-Nitrophenyl-octyl ether & Octadecylamine \\
S1:2 & S2:2 & & Oleyl alcohol \\
S1:3 & S2:3 & & Methyltrioctylammonium chloride \\
S1:4 & S2:4 & & Oleic acid \\
S1:5 & S2:5 & Tris(2-ethyl-hexyl) & Octadecylamine \\
& & phosphate & \\
S1:6 & S2:6 & & Oleyl alcohol \\
S1:7 & S2:7 & & Methyltrioctylammonium chloride \\
S1:8 & S2:8 & & Oleic acid \\
S1:9 & S2:9 & Bis(1-butylpentyl adipate) & Octadecylamine \\
S1:10 & S2:10 & & Oleyl alcohol \\
S1:11 & S2:11 & & Methyltrioctylammonium chloride \\
S1:12 & S2:12 & & Oleic acid \\
S1:13 & S2:13 & Dibutyl sebacate & Octadecylamine \\
S1:14 & S2:14 & & Oleyl alcohol \\
S1:15 & S2:15 & & Methyltrioctylammonium chloride \\
S1:16 & S2:16 & & Oleic acid \\
S1:17 & S2:17 & Bis(2-ethylhexyl)phthalate & Octadecylamine \\
S1:18 & S2:18 & & Oleyl alcohol \\
S1:19 & S2:19 & & Methyltrioctylammonium chloride \\
S1:20 & S2:20 & & Oleic acid \\
& &
\end{tabular}

similarly to previous works [31]. The sensor membranes contained a lipid additive $(\approx 3 \%)$, a plasticizer $(\approx 65 \%)$ and high molecular weight polyvinyl chloride (PVC, $\approx 32 \%$ ) (as shown in Table 1). All reagents were from Fluka (minimum purity $\geq 97 \%$ ). The type of sensors and polymeric membrane compositions (relative percentage of additive, plasticizer and PVC) were chosen considering literature data [32], being used 4 lipid additives and 5 plasticizers. For this type of lipid membranes suitable signal stability over time (\% RSD <5\%) and repeatability $(0.5 \%<\%$ RSD $<15 \%)$ towards the basic standard taste compounds (sweet, acid, bitter, salty and umami) have been previously reported [32]. Moreover, it is known that lipid polymeric membranes may interact with taste substances via electrostatic or hydrophobic interactions [33]. As in previous works [31] each sensor was coded with a letter $\mathrm{S}$ (for sensor) followed by a code for the sensor array (1: or 2:) and the number of the membrane 1-20, corresponding to different combinations of plasticizer and additive used. When needed, an extra uppercase letter was used for referring to the simultaneous electrochemical analysis of table olives ("O_") or brine solutions ("B_").

\subsection{E-tongue response towards typical table olives organoleptic defects}

The E-tongue capability for perceiving organoleptic defects due to abnormal fermentation (i.e., butyric, putrid and zapateria sensations) or other sensory defects (like winey-vinegary and musty), was studied firstly using reference solutions (depending on the availability of the standard compounds referred in the official regulation of IOC [6]) or using real table olive samples or brine solutions, for which a single intense defect was identified by the sensory panel (with minimum intensity of 8 in a 11-intensity scale grade).

\subsubsection{Standard solutions mimicking usual table olives sensory defects}

As recommended by IOC [6], standard solutions were used to mimic the usual sensory defects detected in table olives (and brine solutions) due to abnormal fermentations According to the referred regulation, and as shown in Table 2, putrid defect should be mimicked using 2- mercaptoethanol (Acros Organics, 99\%), butyric defect can be described using n-butyric acid (Acros Organics, > 99\%) and zapateria defect may be simulated using cyclohexanecarboxylic acid (Acros Organics, >98\%). Although for sensory panel training, IOC [6]
Table 2

Standard solutions of table olives' sensory defects due to abnormal fermentation (according to IOC [6]): defect, standard compound, concentration range and organoleptic intensity levels.

\begin{tabular}{llll}
\hline $\begin{array}{l}\text { Defect } \\
\text { attributes }\end{array}$ & $\begin{array}{l}\text { Standard compound } \\
\text { (chemical formula) }\end{array}$ & $\begin{array}{l}\text { Concentrations } \\
\text { (g/L) }\end{array}$ & $\begin{array}{l}\text { Sensory } \\
\text { intensity } \\
(\mathbf{1}-\mathbf{1 1} \\
\text { scale) }\end{array}$ \\
\hline Butyric & $\begin{array}{l}\text { n-butyric acid }\left(\mathrm{C}_{4} \mathrm{H}_{8} \mathrm{O}_{2}\right) \\
\text { 2- Mercaptoehtanol } \\
\text { Putrid }\end{array}$ & 0.5 and 1.0 & 3.5 and 7 \\
Zapateria & $\begin{array}{l}\left.\mathrm{C}_{2} \mathrm{H}_{6} \mathrm{OS}\right) \\
\text { cyclohexanecarboxylic } \\
\text { acid }\left(\mathrm{C}_{6} \mathrm{H}_{11} \mathrm{CO}_{2} \mathrm{H}\right)\end{array}$ & 0.1 and 1.0 & 2.5 and 8 \\
& & & 4 and 9 \\
\hline
\end{tabular}

recommends only the evaluation of each organoleptic defect at two concentration levels, which correspond to known sensory intensities (Table 2), in this work 5 concentrations of each standard solution were prepared and used, which included the recommended range: 0.05-2 g/ L for 2- mercaptoethanol; 0.25-2 g/L for n-butyric acid; and 0.075$2 \mathrm{~g} / \mathrm{L}$ for cyclohexanecarboxylic acid. These standard solutions were made using commercial mineral water, aiming simulating real solutions assessed by the sensory panel (for which deionised or distilled water cannot be used). For each electrochemical assay, $20 \mathrm{~mL}$ of each standard solution were used.

\subsubsection{Reference table olives and brine solutions identified with a single intense sensory defect}

The E-tongue performance towards typical sensory defects of tables olives was further evaluated by using, as previously mentioned, selected table olive samples (and the respective brine solutions), for which a unique/predominant defect was identified by the sensory panel (with minimum median DPP intensity of 8 in a 11-intensity scale grade). It should be remarked that these samples were only used for this purpose. For this study it was possible to identify 3 table olive samples containing a single/predominant very intense organoleptic defect, two of them related to the abnormal fermentation (butyric or zapateria) and third to other defects category (winey-vinegary), according to the IOC classification [6]. So, for the brine solutions, 5 brine samples were chosen according to the sensory panel evaluation, corresponding to single highly intense organoleptic defects: butyric, putrid, zapateria, winey-vinegary or musty single defects (the first 3 related to abnormal fermentations and the last 2 belonging to the other defects category).

For the E-tongue assays, each table olive paste was first ginned and then crushed using a shredder knives Moulinex equipment, yielding a fine-grained paste. Before the electrochemical analysis, each paste was diluted with commercial mineral water resulting in 5 different diluted paste proportions (from $9 \mathrm{~g}$ of table olive paste with $6 \mathrm{~mL}$ mineral water to $5 \mathrm{~g}$ of table olive paste with $10 \mathrm{~mL}$ mineral water).

Regarding the 5 different brine solutions with sensory defects, each liquid sample was initially analyzed without dilution $(20 \mathrm{~mL})$ and then other 4 diluted brine solutions (using mineral water) were also assayed (ranging from $16 \mathrm{~mL}$ of brine solution with $4 \mathrm{~mL}$ of mineral water to $4 \mathrm{~mL}$ brine in $16 \mathrm{~mL}$ mineral water), in order to achieve five concentrations/intensity levels of each single defect.

For analysis, each diluted olive paste or brine solution was placed in transparent plastic pots properly coded.

\subsubsection{Table olive commercial samples and brine solutions}

The 44 table olive samples were also electrochemically analyzed (both olives and the respective brine solutions) using the E-tongue device. As previously described, olives were prior reduced to a paste. First, olive stones were removed and then the pulp was crushed using shredder knives Moulinex equipment. The process turned out into a 
fine-grained paste, which was diluted using mineral water ( $9 \mathrm{~g}$ of table olive paste diluted in $6 \mathrm{~mL}$ mineral water). Each diluted paste was placed into transparent plastic pots properly coded. For the brine solutions, $20 \mathrm{~mL}$ of each sample were also placed in transparent plastic pots properly coded.

\subsubsection{E-tongue assays}

All samples were analyzed at $20^{\circ} \mathrm{C}$ (room temperature) under agitation using a magnetic stirrer (Velp Scientifica). The E-tongue was immersed directly in each sample and, after 5-min of stabilization period, the potentiometric signal profile from the 40 lipid membranes of the multi-sensor system was recorded. During the signal stabilization period, the system allowed monitoring each of the 40 lipid sensor signals (each $10 \mathrm{~s}$ ) and so, enabled the visualization of the potentiometric signal changes versus time.

\subsection{Statistical analysis}

Linear discriminant analysis (LDA) was used as a supervised pattern recognition method to infer about the capability of the Etongue to correctly classify the standards recommended by IOC [6] of negative attributes related to abnormal fermentations (i.e., butyric, putrid and zapateria). It was also used to classify single predominant organoleptic defects (abnormal fermentation and other defects according to IOC [6]) identified by the trained sensory panel in table olives samples and respective brine solutions. The best subsets of $K$ independent predictors among the 40 E-tongue potentiometric signals recorded were chosen using a meta-heuristic simulated annealing (SA) variable selection algorithm [34-36]. The SA algorithm selects the optimal conditions based on the assumptions of the annealing physic process using an iterative procedure. The algorithm searches for a global minimum that optimizes a system with $k(\leq K)$ variables. In each iteration, the solutions of the current and the new subsets of $k$ variables are compared using the tau2 quality criterion, which is a measure of the goodness of fitting. A new solution is randomly selected in the neighborhood of the current solution, being selected if it gave a better result than the initial one. In general, 10,000 attempts are used to select the best subset of variables (best model), starting the process of selecting the best subsets of variables on each trial, thus ensuring a greater confidence in finding a true optimal solution [34].

To evaluate the LDA classification model, first a leave-one-out cross-validation (LOO-CV) procedure was applied. This process may lead to over-optimistic results although, it has proven to be an adequate procedure when the number of samples is low [31,37]. To minimize this risk, a repeated $K$-fold cross-validation (repeated $K$-fold-CV) strategy was also applied to the sub-sets of sensors selected by the SA algorithm. For this purpose, data is randomly, more than once depending on the number of repeats, each time divided into $K$ folds of approximately equal size. Each of the folds is left out in turn and the other $K-1$ folds are used to train the model. The held out fold is predicted and these predictions are summarized according to a performance measure like the sensitivity (e.g., the percentage of correct classifications). The $K$ estimates are averaged to get the overall resampled estimate [38]. In this work the number of $K$-folds was set equal to 4, enabling the random formation of internal validation subsets (for each sensory group) with $25 \%$ of initial data allowing bias reduction, being the procedure repeated 10 times. The repeated $K$-fold$\mathrm{CV}$ procedure was implemented to reduce the uncertainty of the estimates, by evaluating the predictive performance of the models established using $4 \times 10$ random sub-sets for internal validation (i.e., 40 total resamples). To normalize the weight of each variable in the final linear classification model, variable scaling and centering procedures were evaluated. The classification performance of each LDA model was graphically evaluated using: a 2-D plot of the two first discriminant functions with the decision boundary lines between classes; and a plot of the smooth density estimate of each class data for the first discriminant function, which allowed perceiving its influence in classes' separation. Statistical analysis was performed using the Subselect [34] and MASS [39] packages of the open source statistical program $\mathrm{R}$ (version 2.15.1) at a significance level of $5 \%$.

\section{Results and discussion}

\subsection{Table olives sensory analysis and trade category classification}

Each table olive sample was evaluated regarding the negative sensory attributes by 8 panelists trained according to the IOC regulations [6]. The type and intensity level of the negative attributes (i.e., organoleptic defects related to abnormal fermentations or other sensory defects) perceived in the 44 independent table olive samples (olives and respective brine solutions) were evaluated by each panelist. According to the sensory analysis, in 7 table olive samples (olives and brine solutions) none organoleptic defect could be perceived (DPP=1), being classified as extra without organoleptic defects (extra_wd). For the other 37 table olive samples and/or brine solutions, the sensory panel identified at least one of five organoleptic defects (i.e., butyric, putrid, zapateria, musty and/or winey-vinegary). Based on the median intensity level of the DPP (as described in Section 2.1) table olives samples were classified as follows: 11 samples as extra $(1.5 \leq \mathrm{DPP} \leq 3)$, 13 samples as $1^{\text {st }}$ choice $(3.5 \leq \mathrm{DPP} \leq 4.5), 7$ samples as $2^{\text {nd }}$ choice $(5 \leq \mathrm{DPP} \leq 7.0)$ and the remaining 6 samples as olives that may not be sold as table olives $(7.5 \leq \mathrm{DPP} \leq 10)$ (Table 3$)$.

\subsection{E-tongue evaluation of negative sensory attributes}

The capability of the potentiometric E-tongue to differentiate organoleptic defects of table olives relate with the abnormal fermentations (i.e., butyric, putrid and zapateria sensations) or other sensory defects commonly found (like winey-vinegary and musty) was evaluated using standard solutions (of chemical reference compounds recommended by IOC [6]) or using real samples (table olives and brine solutions) identified as reference defect samples by the sensory panel (i.e., samples for which a single intense organoleptic defect, with a minimum median value equal or greater than 8 , was perceived).

\subsubsection{Reference standard solutions mimicking negative organoleptic attributes}

The E-tongue discrimination performance was evaluated using aqueous standard solutions of 2-mercaptoethanol $\left(\mathrm{C}_{2} \mathrm{H}_{6} \mathrm{OS}\right)$, n-butyric acid $\left(\mathrm{C}_{4} \mathrm{H}_{8} \mathrm{O}_{2}\right)$ and ciclohexanecarboxylic acid $\left(\mathrm{C}_{6} \mathrm{H}_{11} \mathrm{CO}_{2} \mathrm{H}\right)$ [6], prepared with commercial mineral water to mimic the conditions used during sensory panel training/evaluation, which concentrations included those recommended by IOC [6]. For the three standard studied, the potentiometric signals recorded with the 20 different lipid-polymeric membranes (S1:1 to $\mathrm{S} 1: 20$, mixtures of 4 additives $\times 5$ plasticizers) and the respective 20 replicas (S2:1 to S2:20) showed a linear (positive or negative) dependency with the decimal logarithm of the concentration. For 2-mercaptoethanol (putrid sensation: 0.05-2 g/L) the signals varied from $-0.25 \mathrm{~V}$ to $+0.22 \mathrm{~V}$ and sensitivities (i.e., slope values) of $-156 \mathrm{mV} /$ decade to $-10 \mathrm{mV} /$ decade were found (correlation coefficient: -0.9002 to -0.9993$)$. For n-butyric acid solutions (that mimic butyric negative organoleptic sensation), the signals varied from $-0.11 \mathrm{~V}$ to $+0.22 \mathrm{~V}$, showing both negative and positive sensitivities (from $-287 \mathrm{mV} /$ decade to $-67 \mathrm{mV} /$ decade, $0.923 \leq R^{2} \leq 0.999$; and, from $+14 \mathrm{mV} /$ decade to $+197 \mathrm{mV} /$ decade, $0.901 \leq R^{2} \leq 0.998$ ). For standard solutions of ciclohexanecarboxylic acid (used to mimic zapateria negative attribute) the recorded signals varied between $-0.07 \mathrm{~V}$ and $+0.20 \mathrm{~V}$ with sensitivities varying between $+4 \mathrm{mV} /$ decade and $+78 \mathrm{mV} /$ decade $\left(0.908 \leq R^{2} \leq 0.995\right)$. The different electrochemical behaviors found towards each standard chemical compound could be tentatively explained due to differences in the composition of the lipidplasticizer-PVC membranes. Indeed, depending on the type of plasti- 
Table 3

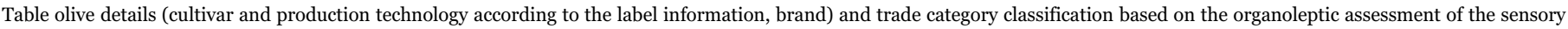
panel (following the IOC regulation [6]).

\begin{tabular}{|c|c|c|c|c|c|}
\hline Sample number & Cultivar $^{\mathrm{a}}$ & Production method $^{\mathrm{b}}$ & Brand & $\mathbf{D P P}^{\mathrm{c}}$ & Trade category classification ${ }^{d}$ \\
\hline 1 & Galega & Natural Fermentation & $\mathrm{A}$ & Putrid & Extra \\
\hline 2 & Galega & Natural Fermentation & B & Winey-vinegary & $1^{\text {st }}$ choice \\
\hline 3 & Galega & Natural Fermentation & $\mathrm{A}$ & Zapateria & Extra \\
\hline 4 & Galega & Natural Fermentation & $\mathrm{E}$ & Winey-vinegary & Extra \\
\hline 5 & Galega & Natural Fermentation & $\mathrm{C}$ & Putrid & $\mathrm{NC}$ \\
\hline 6 & Galega & Natural Fermentation & $\mathrm{C}$ & Winey-vinegary & $1^{\text {st }}$ choice \\
\hline 7 & Galega & Natural Fermentation & $\mathrm{C}$ & Putrid & $2^{\text {nd }}$ choice \\
\hline 8 & Galega & Natural Fermentation & $\mathrm{C}$ & Putrid & $2^{\text {nd }}$ choice \\
\hline 9 & Galega & Natural Fermentation & $\mathrm{C}$ & Putrid & $2^{\text {nd }}$ choice \\
\hline 10 & Galega & Natural Fermentation & $\mathrm{C}$ & Putrid & $1^{\text {st }}$ choice \\
\hline 11 & Galega & Natural Fermentation & $\mathrm{D}$ & Musty & Extra \\
\hline 12 & Galega & Natural Fermentation & $\mathrm{D}$ & Winey-vinegary & $1^{\text {st }}$ choice \\
\hline 13 & Galega & Natural Fermentation & $\mathrm{D}$ & Winey-vinegary & Extra \\
\hline 14 & Galega & Natural Fermentation & $\mathrm{D}$ & Winey-vinegary & $1^{\text {st }}$ choice \\
\hline 15 & Galega & Natural Fermentation & $\mathrm{D}$ & Putrid & $\mathrm{NC}$ \\
\hline 16 & Galega & Natural Fermentation & $\mathrm{A}$ & Butyric & $2^{\text {nd }}$ choice \\
\hline 17 & Galega & Natural Fermentation & $\mathrm{A}$ & Butyric & $1^{\text {st }}$ choice \\
\hline 18 & Galega & Natural Fermentation & A & Zapateria & $1^{\text {st }}$ choice \\
\hline 19 & Galega & Natural Fermentation & $\mathrm{A}$ & Putrid & $1^{\text {st }}$ choice \\
\hline 20 & Galega & Natural Fermentation & $\mathrm{A}$ & Putrid & $1^{\text {st }}$ choice \\
\hline 21 & Galega & Natural Fermentation & $\mathrm{E}$ & Musty & $2^{\text {nd }}$ choice \\
\hline 22 & Galega & Natural Fermentation & $\mathrm{B}$ & Winey-vinegary & $1^{\text {st }}$ choice \\
\hline 23 & Mixed & Natural Fermentation & $\mathrm{F}$ & Zapateria & Extra \\
\hline 24 & Galega & Natural Fermentation & G & Musty & Extra \\
\hline 25 & Mixed & Natural Fermentation & G & Zapateria & Extra \\
\hline 26 & Negrinha de freixo & Natural Fermentation & G & Avinhado & Extra \\
\hline 27 & Mixed & Natural Fermentation & G & Free of defects & Extra_wd \\
\hline 28 & Manzanilla & Spanish-Style & I & Free of defects & Extra_wd \\
\hline 29 & Hojiblanca & Spanish-Style & $\mathrm{H}$ & Free of defects & Extra_wd \\
\hline 30 & Galega & Natural Fermentation & $\mathrm{J}$ & Winey-vinegary & $2^{\text {nd }}$ choice \\
\hline 31 & Mixed & Mix all processes & G & Free of defects & Extra_wd \\
\hline 32 & Gordal & Spanish-Style & M & Free of defects & Extra_wd \\
\hline 33 & Hojiblanca & California-Style & $\mathrm{H}$ & Free of defects & Extra_wd \\
\hline 34 & Hojiblanca & Spanish-Style & $\mathrm{H}$ & Free of defects & Extra_wd \\
\hline 35 & Mixed & Spanish-Style & G & Winey-vinegary & $1^{\text {st }}$ choice \\
\hline 36 & Manzanilla & Natural Fermentation & $\mathrm{L}$ & Winey-vinegary & $1^{\text {st }}$ choice \\
\hline 37 & Empeltre & Natural Fermentation & $\mathrm{N}$ & Musty & $1^{\text {st }}$ choice \\
\hline 38 & Mixed & Natural Fermentation & $\mathrm{K}$ & Putrid & $2^{\text {nd }}$ choice \\
\hline 39 & Mixed & Natural Fermentation & $\mathrm{K}$ & Winey-vinegary & Extra \\
\hline 40 & Galega & Natural Fermentation & $\mathrm{J}$ & Winey-vinegary & Extra \\
\hline 41 & Cobrançosa & Spanish-Style & $\mathrm{O}$ & Butyric & $\mathrm{NC}$ \\
\hline 42 & Cobrançosa & Natural Fermentation & $\mathrm{P}$ & Butyric & $\mathrm{NC}$ \\
\hline 43 & Cobrançosa & Natural Fermentation & $\mathrm{Q}$ & Musty & $\mathrm{NC}$ \\
\hline 44 & Negrinha de freixo & Natural Fermentation & $\mathrm{R}$ & Butyric & $\mathrm{NC}$ \\
\hline
\end{tabular}

a Table olives' cultivar as mentioned in the label.

b Technological method used to produce the table olives according to the label.

${ }^{\mathrm{c}}$ Prevailing negative attribute perceived by the sensory panel in each sample (DPP).

d Table olives trade category classification according to the 4 classes recommended by IOC [6] (based on the median intensity of the defect predominantly perceived, DPP, by the

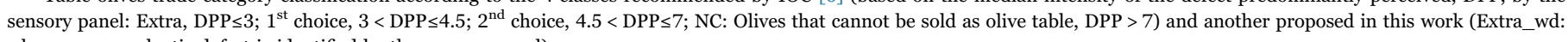
when no organoleptic defect is identified by the sensory panel).

cizer used (which increases solutes absorption by softening the membrane) and additive (lipid substances) applied, the membrane will exhibit different properties depending on the $\mathrm{pH}$, showing either hydrophobic or hydrophilic interactions. Indeed, PVC polymer molecules have internal positive and negative polarities, while plasticizer and additive molecules have polar and non-polar regions. Moreover, since the sensor membrane behaves like a polymer, its permeability depends on the solutes' molecular volume.

The results show that the E-tongue, containing lipid membranes as sensing units, quantitatively respond towards standard solutions mimicking negative organoleptic attributes (butyric, putrid and zapateria), which is reported for the first time since until now, E-tongue were only described as taste sensors for basic taste attributes like acid, bitter, salty, sweet and umami $[32,33,40]$.

Furthermore, the potential use of the E-tongue to qualitatively discriminate the organoleptic negative attributes, usually found in tables olives due to the abnormal fermentation, was also assessed using a supervised multivariate statistical tool (LDA) coupled with a meta-heuristic SA variable selection algorithm. The LDA-SA approach aimed to identify the best sub-set of E-tongue sensors (among the 20 sensors and their replicas), which should comprise the minimum number of independent sensors. The results confirmed the capability of the E-tongue-LDA-SA procedure for discriminating standard solutions mimicking sensory defects. A model with 2 significant functions (P-value < 0.0001 ; explaining $98.4 \%$ and $1.6 \%$ of the original data variability) based on the potentiometric signal profiles of 5 sensors (S1:10, S1:12, S2:8, S2:10 and S2:20; which included oleyl alcohol and oleic acid as additive compounds) was used. The LDA-SA model allowed the correct classification of $100 \%$ of the original grouped data (Fig. 1) and 93\% of the standard solutions for LOO-CV procedure (i.e., 1 solution with zapateria defect was misclassified as putrid defect). As can be inferred from Fig. 1, from the 2-D discrimination plot as well as density distribution plot, the 1st discriminant function almost allows total differentiation of the defect sensations evaluated, with a slight 
$\square$ n-Butyric acid $(0.25$ to $2 \mathrm{~g} / \mathrm{L})$

○ 2-mercaptoethanol (0.05 to $2 \mathrm{~g} / \mathrm{L})$

$\triangle$ Cyclohexanecarboxylic ( 0.075 to $2 \mathrm{~g} / \mathrm{L})$
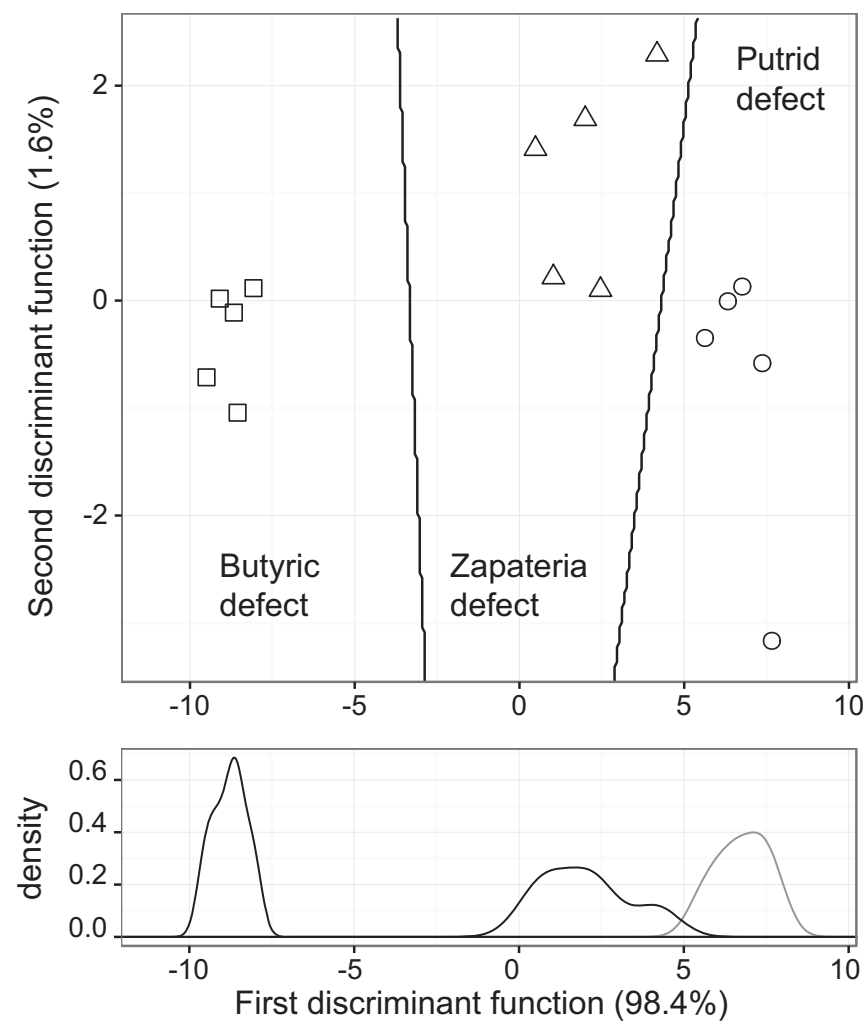

Fig. 1. Discrimination of standard chemical solutions according to the organoleptic defect mimicked and usually perceived during table olives sensory analysis due to the abnormal fermentation, using an E-tongue-LDA-SA model based on the information gathered by 5 sensors of the E-tongue (S1:10, S1:12, S2:8, S2:10 e S2:20): n-butyric acid (0.25-2 g/L; butyric defect); 2-mercaptoethanol (0.05-2 g/L; putrid defect); and ciclohexanecarboxylic acid $(0.075-2 \mathrm{~g} / \mathrm{L}$; zapateria defect).

overplot between zapateria and putrid sensations. Also, it should be remarked that the model included a sensor and its respective replica (S1:10 and S2:10), which signal profiles may be different due to the slight variations in membrane composition and physical properties (transparency and porosity) that may occur when a drop-by-drop technique is used [31]. Moreover, the inclusion of repeated sensors in multivariate models is known to improve model performance [41].

To further test the robustness of the predictive performance of the E-tongue-LDA-SA model to differentiate sensory negative attributes, a repeated $K$-fold-CV procedure ( 4 folds $\times 10$ repetitions) was applied. For the negative attributes, the best predictive classification results were obtained using a model also based on the same 5 sensors that enabled achieving an average correct classification of $97 \pm 8 \%$ (varying from $75 \%$ to $100 \%$ for the 40 evaluations performed during the repeated $K$ fold-CV procedure).

\subsubsection{E-tongue discriminant potential of table olives and brine samples with a single intense defect}

The capability of the E-tongue to discriminate specific very intense organoleptic defects (median DPP intensity $\geq 8$ ) in real samples was evaluated by analyzing tables olives and brine solutions samples, selected according to the sensory evaluation performed by the trained panel. The sensory analysis allowed identifying table olive samples with 3 single highly intense organoleptic defects (butyric, winey-vinegary and zapateria) as well as brine solution samples with 5 single defects (butyric, musty, putrid, winey-vinegary and zapateria). From each table olive or brine sample, 5 sub-samples were obtained with different defect intensities by diluting the initial sample with mineral water (as described in Section 2.4.2), resulting in a total of 15 and 25 independent sub-samples, which were electrochemically analyzed. The potentiometric signals recorded with the E-tongue device varied from $-0.27 \mathrm{~V}$ to $+0.28 \mathrm{~V}$, during the electrochemical assays of table olive aqueous pastes and brine solutions.

For table olives identified with a single negative organoleptic attribute a classification E-tongue-LDA-SA model (2 linear discriminant functions explaining $99.97 \%$ and $0.03 \%$ of the data variability) was established based on the electrochemical signal profiles recorded during the olive paste analysis by 7 E-tongue sensors (S1:9, S1:15, $\mathrm{S} 2: 6, \mathrm{~S} 2: 14, \mathrm{~S} 2: 15, \mathrm{~S} 2: 17$ and S2:20). The results showed that the model was able to correctly classify $100 \%$ of the table olive-paste solutions according to the 3 main defects identified (butyric, wineyvinegary or zapateria), for the original grouped data (Fig. 2A) as well as, for the LOO-CV procedure. Concerning the classification of the reference brine solutions according to each one of the 5 intense sensory defects perceived by the sensory panel (butyric, putrid, zapateria, winey-vinegary and musty), an E-tongue-LDA-SA model (for which the first 2 discriminant significant functions explained $99.7 \%$ and $0.2 \%$ of the data variability) was obtained based on the signal profiles of 10 E-tongue sensors (S1:1, S1:2, S1:16, S1:17, S2:4, S2:7, S2:15, S2:16 and $\mathrm{S} 2: 20$ ). The classification model also enabled the correct classification of $100 \%$ of the brine solutions according to the type of sensory defect for the original grouped data (Fig. 2B) and for the LOO-CV approach. Also, Fig. 2 (2-D discrimination and density distribution plots) pointed out that the 1st discriminant function enabled a full differentiation of the single intense organolpetic defects perceived in real olive and brine samples.

Once again, prediction performance of the E-tongue-LDA-SA models was evaluated using the repeated $K$-fold-CV procedure ( $K=4$ with 10 repeats) trying to minimize the overfitting risk. The results showed that the best E-tongue-LDA-SA models for classifying table olives or brine solutions according to the organolpetic perceived intense defects were based on the same 7 or 10 E-tongue sensors, which allowed achieving, respectively, mean correct classifications of $93 \pm 12 \%$ (sensitivities varying from $67 \%$ to $100 \%$ regarding the 40 split repetitions performed) and $98 \pm 5 \%$ (sensitivities ranging between $83 \%$ and $100 \%$ ). The slightly better predictive results obtained for the brine solutions may be due to the higher difficulty in performing the electrochemical analysis for the table olives, which must be previously converted into aqueous pastes.

\subsection{E-tongue performance as table olives' classification tool according to level of perceived sensory defects}

The E-tongue capability for classifying the table olives according to organoleptic quality levels, established according to IOC guidelines [6], which take into account the perceived intensity of sensory defects, was evaluated using 44 independent commercial table olives and their brine solutions.

So, aqueous pastes of the table olive samples and the respective undiluted brine solutions were electrochemically analyzed (as described in Sections 2.4 .3 and 2.4.4), totalizing 80 sensor signals recorded for each one of the 44 samples described in Table 3. The potentiometric signal data collected (varying from $-0.24 \mathrm{~V}$ to $+0.15 \mathrm{~V}$ ) were used to establish the best E-tongue-LDA-SA model for discriminating the table olives according to IOC quality sensory grades [6] and that proposed in this work: 7 samples classified as extra free of organolpetic defects, 11 samples classified as extra, 13 samples classified as $1^{\text {st }}$ choice, 7 samples classified as $2^{\text {nd }}$ choice and 6 samples classified as olives that may not be sold (Table 3).

An E-tongue-LDA-SA model (2 discriminant functions explained $99.7 \%$ and $0.2 \%$ of the original data variability) was established based on the signal profiles recorded for the brine solutions ( 9 sensors: $\mathrm{S} 1: 2$, S1:4, S1:6, S1:20, S2:7, S2:11, S2:13, S2:15 and S2:20) and the table 

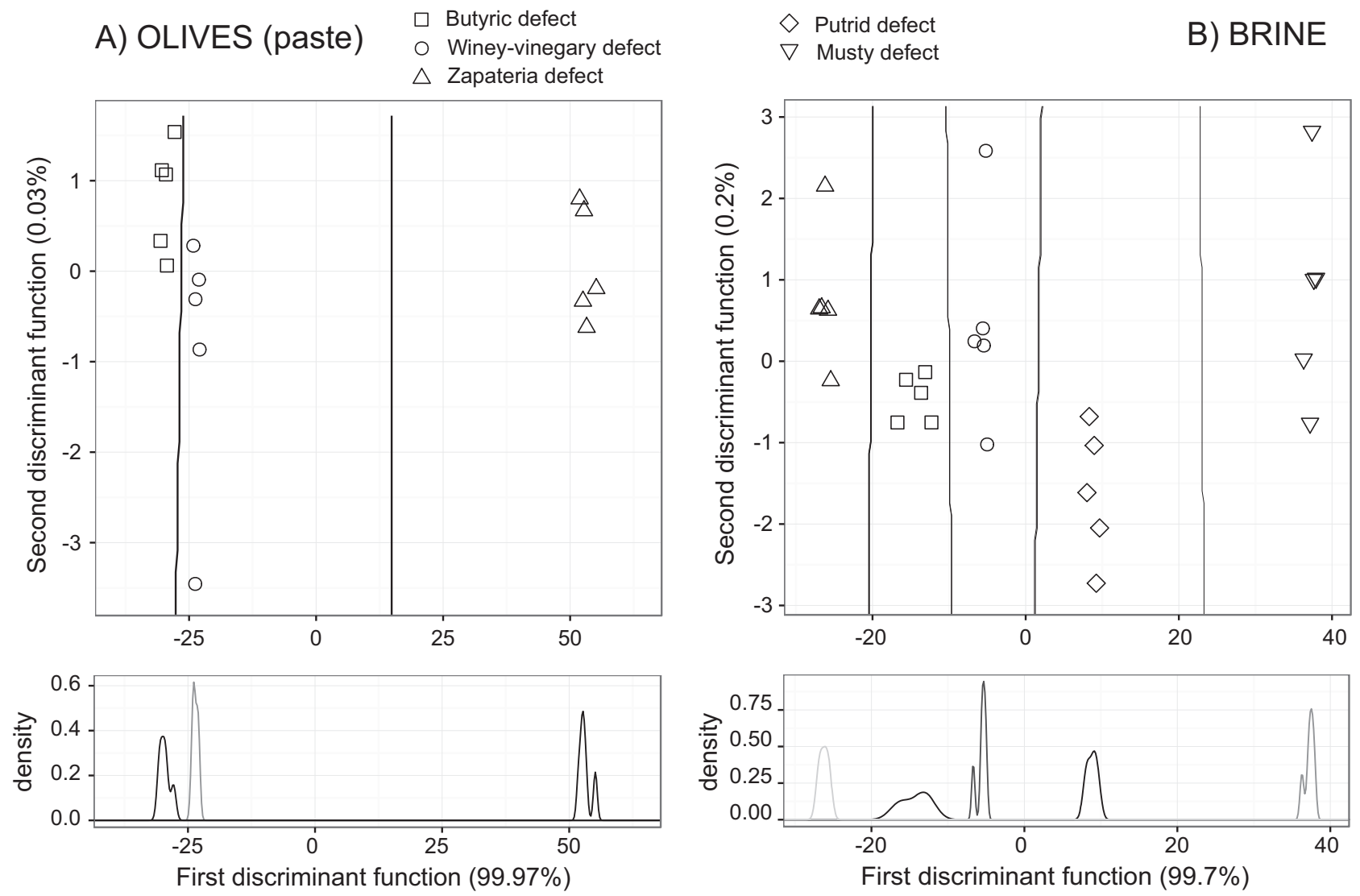

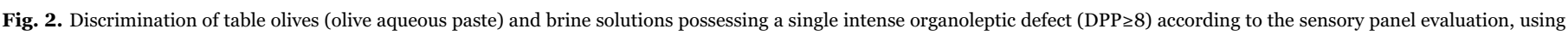

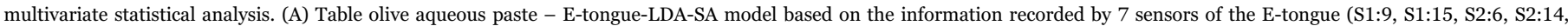

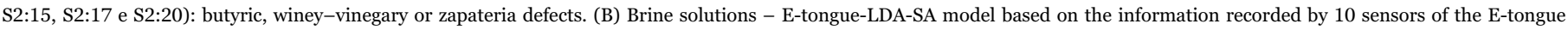
(S1:1, S1:2, S1:16, S1:17, S2:4, S2:7, S2:15, S2:16 e S2:20): butyric, musty, putrid, winey-vinegary or zapateria.

olive aqueous pastes (16 sensors: $\mathrm{S} 1: 4, \mathrm{~S} 1: 10, \mathrm{~S} 1: 11, \mathrm{~S} 1: 13, \mathrm{~S} 1: 16$, $\mathrm{S} 1: 18, \mathrm{~S} 2: 2, \mathrm{~S} 2: 3, \mathrm{~S} 2: 8, \mathrm{~S} 2: 9, \mathrm{~S} 2: 12$ to $\mathrm{S} 2: 14, \mathrm{~S} 2: 16, \mathrm{~S} 2: 17$ and $\mathrm{S} 2: 19)$. As can be seen, the model uses more information from the table olive aqueous pastes analysis compared to that obtained from the brine solutions, which was expected since, even for the panelists, organoleptic defects are more easily perceived in the olives than in brine solutions. It can be inferred that, in general, the sensor sub-set from each matrix is not the same, which could be attributed to the fact that the different defects may be perceived in the olives and brine solutions. Finally, the E-tongue-LDA-SA model allowed the correct classification of $100 \%$ for the original grouped data (Fig. 3) and $97.7 \%$ for the LOOCV procedure (being only 1 sample misclassified). Also, both plots of Fig. 3 show that the 1st discriminant function is sufficient for differentiating the 5 table olives' sensory quality trade levels.

The E-tongue-LDA-SA predictive performance was further assessed using the repeated $K$-fold-CV procedure. The results showed that best predictive E-tongue-LDA-SA models were based on the same 25 signal profiles ( 9 acquired during the brine solutions analysis and the other 16 during the table olive aqueous pastes evaluation) previously described, resulting in a mean correct classification of $86 \pm 9 \%$ (sensitivities varying from $73 \%$ to $100 \%$ ). It is important to emphasize that the results are quite satisfactory taking into account the heterogeneity of the 44 commercial table olives studied in this work (18 different brands; 7 different olive cultivars plus olive blends; and, produced using 3 types of technological processes, aromatized or not with spices, as described in Section 2.1 and Table 3). Furthermore, the samples evaluated showed a high sensory complexity considering that more than one sensory defect could be perceived by the panelists as well as basic gustatory attributes (e.g., salty, bitter and acid). Therefore, the overall average predictive classification performance obtained with the E-tongue can be seen as a promising tool for table olives sensory groups' classification.

\section{Conclusions}

In this work it was demonstrated the feasibility of applying a potentiometric E-tongue (with lipidic cross-sensitivity polymeric membranes) in combination with chemometric tools, for the successful discrimination of negative organoleptic defects (standard solutions and reference real samples) usually detected in table olives and their brine solutions; as well as, to satisfactorily classify this well appreciated food product according to commercial trade categories, recognized by the International Olive Council.

The predictive satisfactory classification performance achieved with the data fusion of the electronic tongue data and linear discriminant analysis, pointed out its possible practical application for table olives evaluation according to negative organoleptic attributes, which could be used as a helpful tool by trained sensory panels. Also, the overall satisfactory results reported show that this electrochemical based approach may be used as a device for verifying the sensory quality of table olives and so, contributing to enhancing consumers' confidence and ensuring that the price paid really reflects the product actual organoleptic quality.

Finally, this study is a proof-of-concept, expanding the applicability range of this type of electrochemical device (electronic tongue) as taste sensors from the known olive oil analysis towards other relevant olive derived products. 

$\nabla$ Extra_WD
$\square$ 1st choice
$\triangle$ Extra
○ 2nd choice
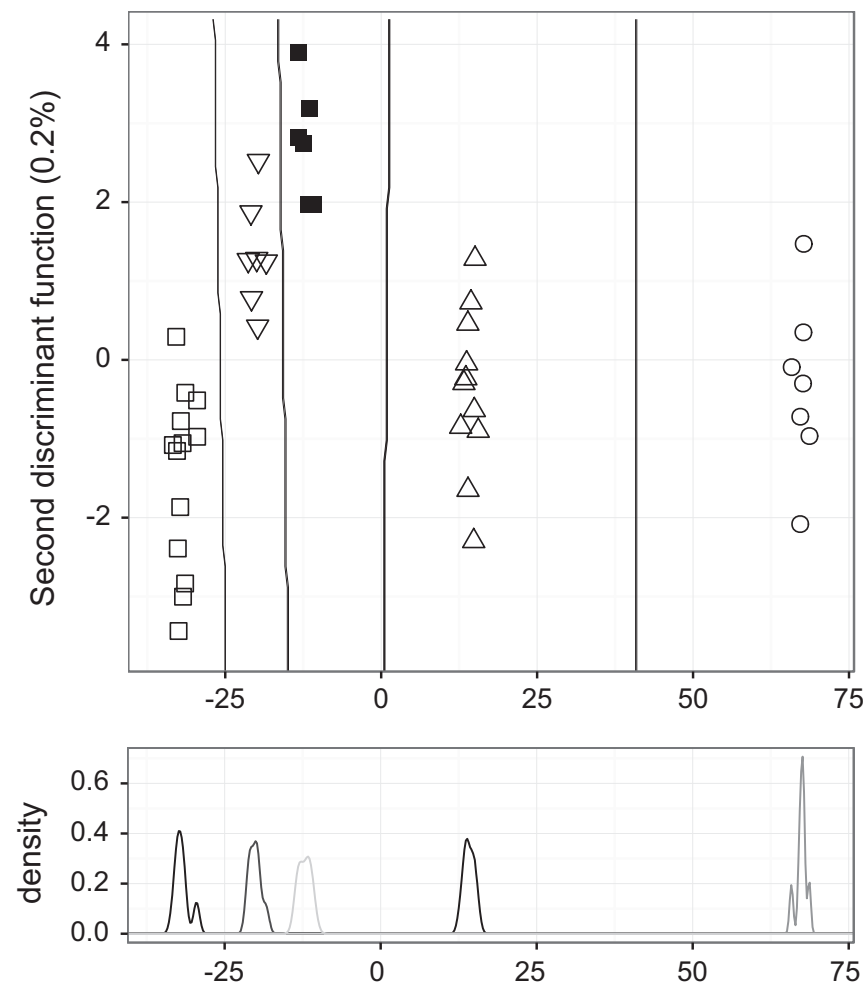

First discriminant function $(99.97 \%)$

Fig. 3. Discrimination of table olives according to their commercial category [6] using an E-tongue-LDA-SA model based on the information of 25 signal potentiometric profiles recorded by the E-tongue during the olive pastes and brine solutions simultaneous analysis: signals from 16 sensors regarding olive paste analysis (O_S1:4, O_S1:10, O_S1:11, O_S1:13, O_S1:16,O_S1: 18,O_S2:2, O_S2:3, O_S2:8, O_S2:9, O_S2:12, O_S2:13, O_S2:14, O_S2:16, O_S2:17 and O_S2:19) and signals from 9 sensors regarding brine solution analysis (B_S1:2, B_S1:4, B_S1:6, B_S1:20, B_S2:7, B_S2:11, B_S2:13, B_S2:15 and B_S2:20). The classification uses the trade categories defined by IOC [6], based on the median intensity of the predominant defect perceived by the sensory panel (extra, $1^{\text {st }}$ choice, $2^{\text {nd }}$ choice and that cannot be sold as table olives) plus an additional category proposed in this work (extra without defects).

\section{Compliance with ethics requirements}

\section{Conflict of interest}

Ítala M. G. Marx declares that she has no conflict of interest. Nuno Rodrigues declares that he has no conflict of interest. Luís G. Dias declares that he has no conflict of interest. Ana C.A. Veloso declares that she has no conflict of interest. José A. Pereira declares that he has no conflict of interest. Deisy A. Drunkler declares that she has no conflict of interest. António M. Peres declares that he has no conflict of interest.

\section{Ethical approval}

This article does not contain any studies with human participants or animals performed by any of the authors.

\section{Informed consent}

Not applicable.

\section{Acknowledgments}

This work was financially supported by Project POCI-01-0145FEDER-006984 - Associate Laboratory LSRE-LCM and by Project UID/QUI/00616/2013 - CQ-VR both funded by FEDER - Fundo Europeu de Desenvolvimento Regional through COMPETE2020 Programa Operacional Competitividade e Internacionalização (POCI) - and by national funds through FCT - Fundação para a Ciência e a Tecnologia, Portugal. Strategic funding of UID/BIO/04469/2013 unit is also acknowledged. Nuno Rodrigues thanks FCT, POPH-QREN and FSE for the Ph.D. Grant (SFRH/BD/104038/2014).

\section{References}

[1] B. Lanza, Chapter 16 - Nutritional and sensory quality of table olives, in: I. Muzzalupo (Ed.)Olive Germplasm - The Olive Cultivation, Table Olive and Olive Oil Industry in Italy, InTech, 2012, pp. 343-372 http://www.intechopen.com/ books/olive-germplasm-the-olive-cultivation-table-olive-and-olive-oil-industry-initaly/nutritional-and-sensory-quality-of-table-olives.

[2] D. Boskou, S. Camposeo, M.L. Clodoveo, Chapter 8 - Table olives as sources of bioactive compounds, in: D. Boskou (Ed.)Olives and Olive Oil Bioactive Constituents, AOCS Press, Urbana, IL, USA, 2015, pp. 217-259.

[3] S. Kailis, D. Harris, Chapter 4 - Table olive processing: general aspects, in: S. Kailis, D. Harris (Eds.), Producing Table Olives, Landlinks Press, CSIRO Publishing, Collingwood, Australia, 2007, pp. 131-189.

[4] Codex Alimentarius Commission, Codex Standard for Table Olives, Codex Stan, 1987, 66-1981. (Rev. 1-1987)

[5] International Olive Oil Council, Trade Standards Applying to Table Olives, IOC/ OT/NC no. 1. International Olive Oil Council, Madrid, Spain, 2004

[6] IOC, Conselho Oleícola Internacional, Method For The Sensory Analysis of Table Olives, 2011. 〈http://www.internationaloliveoil.org/estaticos/view/224-testingmethods $\rangle$ (accessed 10.02.16)

[7] A. Hurtado, C. Reguant, A. Bordons, N. Roze, Influence of fruit ripeness and salt concentration on the microbial processing of Arbequina table olives, Food Microbiol. 26 (2009) 827-833.

[8] M. Aponte, V. Ventorino, G. Blaiotta, G. Volpe, V. Farina, G. Avellone, C.M. Lanza, G. Moschetti, Study of green Sicilian table olive fermentation through microbiological, chemical and sensory analyses, Food Microbiol. 27 (2010) 162-170.

[9] J.M. Moreno-Baquero, J. Bautista-Gallego, A. Garrido-Fernández, A. López-López, Mineral and sensory profile of seasoned cracked olives packed in diverse salt mixtures, Food Chem. 138 (2013) 1-8.

[10] C.L. Randazzo, A. Todaro, A. Pino, I. Pitino, O. Corona, A. Mazzaglia, C. Caggia Giarraffa, G. di Spagna, Naturally fermented table olives: effect of starter and probiotic cultures on chemical, microbiological and sensory traits, Food Res. Int. 62 (2014) 1154-1164.

[11] M. Alves, E. Esteves, C. Quintas, Effect of preservatives and acidifying agents on the shelf life of packed cracked green table olives from Maçanilha cultivar, Food Packag. Shelf Life 5 (2015) 32-40.

[12] A. Martorana, A. Alfonzo, L. Settanni, O. Corona, F. La Croce, T. Caruso, G. Moschetti, N. Francesca, An innovative method to produce green table olives based on "pied de cuve" technology, Food Microbiol. 50 (2015) 126-140.

[13] F. Rodríguez-Gómez, A. López-López, V. Romero-Gil, F.N. Arroyo-López, J.M. Moreno-Baquero, A. Garrido-Fernández, P. García-García, Effect of postfermentation storage on Spanish-style green Manzanilla olives, LWT - Food Sci. Technol. 57 (2014) 789-793.

[14] M. De Angelis, D. Campanella, L. Cosmai, C. Summo, C.G. Rizzello, F. Caponio, Microbiota and metabolome of un-started and started Greek-type fermentation of Bella di Cerignola table olives, Food Microbiol. 52 (2015) 18-30.

[15] M. Campus, P. Sedda, E. Cauli, F. Piras, R. Comunian, A. Paba, E. Daga, S. Schirru, A. Angioni, R. Zurru, G. Bandino, Evaluation of a single strain starter culture, a selected inoculum enrichment, and natural microflora in the processing of Tonda di Cagliari natural table olives: impact on chemical, microbiological, sensory and texture quality, LWT - Food Sci. Technol. 64 (2015) 671-677.

[16] V.A. Blana, N. Polymeneas, C.C. Tassou, E.Z. Panagou, Survival of potential probiotic lactic acid bacteria on fermented green table olives during packaging in polyethylene pouches at 4 and $20^{\circ} \mathrm{C}$, Food Microbiol. 53 (2016) 71-75.

[17] B. Lanza, F. Amoruso, Sensory analysis of natural table olives: relationship between appearance of defect and gustatory-kinaesthetic sensation changes, LWT - Food Sci. Technol. 68 (2016) 365-372.

[18] M. Cano-Lamadrid, F. Hernández, M. Corell, F. Burló, P. Legua, A. Moriana, Á.A. Carbonell-Barrachina, Antioxidant capacity, fatty acids profile, and descriptive sensory analysis of table olives as affected by deficit irrigation, J. Sci. Food Agric. (2016). http://dx.doi.org/10.1002/jsfa.7744.

[19] A. Cortés-Delgado, A.H. Sánchez, A. de Castro, A. López-López, V.M. Beato, A. Montaño, Volatile profile of Spanish-style green table olives prepared from different cultivars grown at different locations, Food Res. Int. 83 (2016) 131-142.

[20] E. Ramírez, P. García, M. Brenes, C. Romero, Evaluation of chemical components of debittered olives undergone preservation and polyphenol oxidation, Int. J. Food Sci. Technol. 51 (2016) 1674-1679.

[21] A. López-López, J. Bautista-Gallego, J.M. Moreno-Baquero, A. Garrido-Fernández, Fermentation in nutrient salt mixtures affects green Spanish-style Manzanilla table 
olive characteristics, Food Chem. 211 (2016) 415-422.

[22] E.Z. Panagou, N. Sahgal, N. Magan, G.-J.E. Nychas, Table olives volatile fingerprints: potential of an electronic nose for quality discrimination, Sens. Actuators B 134 (2008) 902-907.

[23] E.Z. Panagou, C.C. Tassou, Changes in volatile compounds and related biochemical profile during controlled fermentation of cv. Conservolea green olives, Food Microbiol. 23 (2006) 738-746.

[24] T. Navarro, C. de Lorenzo, R.A. Perez, SPME analysis of volatile compounds from unfermented olives subjected to thermal treatment, Anal. Bioanal. Chem. 379 (2004) 812-817.

[25] E. Borràs, J. Ferré, R. Boqué, M. Mestres, L. Aceña, A. Calvo, O. Busto, Olive oil sensory defects classification with data fusion of instrumental techniques and multivariate analysis (PLS-DA), Food Chem. 203 (2016) 314-322.

[26] M.L. Rodríguez-Méndez, C. Apetrei, J.A. de Saja, Chapter 57 - Electronic tongues purposely designed for the organoleptic characterization of olive oils, in: V.R. Preedy, R.R. Watson (Eds.), Olives and Olive Oil in Health and Disease Prevention, Academic Press, London, 2010, pp. 525-532.

[27] C. Apetrei, M. Ghasemi- Vernamkhasti, I.M. Apetrei, Chapter 27 - Olive oil and combined electronic nose and tongue, in: M.L. Rodríguez-Méndez (Ed.)Electronic Noses and Tongues in Food Science, Academic Press, London, 2016, pp. 277-289.

[28] M. Casale, P. Zunin, M.E. Cosulich, E. Pistarino, P. Perego, S. Lanteri, Characterisation of table olive cultivar by NIR spectroscopy, Food Chem. 122 (2010) 1261-1265.

[29] A. Sousa, S. Casal, A. Bento, R. Malheiro, M.B.P.P. Oliveira, J.A. Pereira, Chemical characterization of "alcaparras" stoned table olives from Northeast Portugal, Molecules 16 (2011) 9025-9040.

[30] H. Galán-Soldevilla, P.R. Pérez-Cacho, J.A.H. Campuzano, Determination of the characteristic sensory profiles of Aloreña table-olive, Grasas Aceites 64 (2013)
$442-452$.

[31] L.G. Dias, A. Fernandes, A.C.A. Veloso, A.A.S.C. Machado, J.A. Pereira, A.M. Peres, Single-cultivar extra virgin olive oil classification using a potentiometric electronic tongue, Food Chem. 160 (2014) 321-329.

[32] L.G. Dias, A.M. Peres, A.C.A. Veloso, F.S. Reis, M. Vilas Boas, A.A.S.C. Machado, An electronic tongue taste evaluation: identification goat milk adulterations with bovine milk, Sens. Actuators B 136 (2009) 209-217.

[33] Y. Kobayashi, M. Habara, H. Ikezazki, R. Chen, Y. Naito, K. Toko, Advanced taste sensors based on artificial lipids with global selectivity to basic taste qualities and high correlation to sensory scores, Sensors 10 (2010) 3411-3443.

[34] J. Cadima, J.O. Cerdeira, M. Minhoto, Computational aspects of algorithms for variable selection in the context of principal components, Comput. Stat. Data Anal. 47 (2004) 225-236.

[35] S. Kirkpatrick, C.D. Gelatt, M.P. Vecchi, Optimization by simulated annealing, Science 220 (1983) 671-680.

[36] D. Bertsimas, J. Tsitsiklis, Simulated annealing, Stat. Sci. 8 (1993) 10-15.

[37] J.M. Gutiérrez, Z. Haddi, A. Amari, B. Bouchikhi, A. Mimendia, X. Cetó, M. del Valle, Hybrid electronic tongue based on multisensor data fusion for discrimination of beers, Sens. Actuators B 177 (2013) 989-996.

[38] M. Kuhn, K. Johnson, Applied Predictive Modeling, Springer Science Business Media, New York, USA, 2013.

[39] W.N. Venables, B.D. Ripley, Modern Applied Statistics with S (Statistics and Computing), 4th ed., Springer, New York, USA, 2002.

[40] A.C.A. Veloso, L.G. Dias, N. Rodrigues, J.A. Pereira, A.M. Peres, Sensory intensity assessment of olive oils using an electronic tongue, Talanta 146 (2016) 585-593.

[41] D.P.A. Correia, J.M.C.S. Magalhães, A.A.S.C. Machado, Array of potentiometric sensors for simultaneous analysis of urea and potassium, Talanta 67 (2005) $773-782$. 